\title{
Certificación y Recertificación de Especialistas en Cirugía General. Una mirada desde CONACEM*
}

\author{
Dr. JOSÉ M. LÓPEZ M. ${ }^{1}$
}

1 Presidente de la Corporación Nacional Autónoma de Certificación de Especialidades Médicas (CONACEM). Santiago, Chile.

\begin{abstract}
Certification of surgery specialists in Chile by the autonomous corporation for certification of medical specialties
\end{abstract}

Since 1984, 12.294 different medical specialists have been certified in Chile by the Autonomous Corporation for Certification of Medical Specialties (whose Spanish acronym is CONACEM). General Surgery certification started in 1984, approving 1.088 candidates thus far. Certification can be obtained in three ways. Seventy-two percent of applicants were approved by a reputable university specialization program. Twentythree percent of applicants fulfilled the requirements of a five years practical training program and $5 \%$ were approved in a training program from another country which was validated in Chile. There are 13 university training programs for surgery specialty, lasting three years each. These programs can receive a total of 223 students per year. Thirty-one percent of these programs are not carried out in Santiago. Applicants, who have not completed a certified university training program, must go through a five days practical examination. Since 2002 a written test was added, whose approval is a requisite to gain access to the practical examination. Eighty-nine percent of applicants have approved the theoretical test. Certifications last 10 years initially and seven years, thereafter. When certifications expire, a recertification mechanism is required, whose requirements are informed. According to the current legislation, certifications must be done by recognized accrediting agencies. CONACEM has been accepted by the authority and its definitive legal recognition should be a reality soon.

Key words: Accreditation, certification, surgery, Chile.

\section{Resumen}

Desde 1984 la Corporación Autónoma de Certificación de Especialidades Médicas (CONACEM) ha certificado 12.294 especialistas médicos en Chile. La disciplina de Cirugía General inició su certificación en 1984, habiendo reconocido hasta la fecha a 1.088 especialistas. Para la certificación existen tres vías posibles:

a) haber aprobado un Programa de Formación Universitario Acreditado, lo que hizo el $72 \%$ de los postulantes;

b) cumplir los requisitos de Adiestramiento en Práctica durante 5 años, lo que hizo un $23 \%$ de los postulantes.

*Recibido el 5 de julio de 2013 y aceptado para publicación el 2 de agosto de 2013.

El autor no refiere conflictos de interés.

Correspondencia: Dr. José M. López M.

Esmeralda $678,3^{\circ}$ piso interior, Santiago, Chile.

conacem@entelchile.net 
c) haber sido formado en el extranjero de acuerdo a programas convalidados. Existen 13 programas universitarios acreditados de 3 años de duración, con un cupo total anual de 223 alumnos, de los cuales el $69 \%$ se desarrolla en Santiago y el resto en provincias. Salvo los médicos aprobados en los Programas Universitarios acreditados, los postulantes requieren dar un examen práctico; desde 2002 se ha agregado un examen escrito, cuya aprobación es requisito para dar el práctico, y que ha resultado tener buena discriminación $(11 \%$ de rechazo en el período de 11 años de existencia). Las certificaciones dadas hoy, tienen una duración de 10 años al inicio y de 7 años posteriormente. La caducidad de las certificaciones está aparejada al proceso de recertificación, para lo cual se discuten las razones que lo avalan; se presentan los requisitos y tabla de créditos a cumplir, elaboradas por CONACEM. La legislación vigente y en proceso, requiere el reconocimiento de entidades certificadoras, concurso en el cual ha sido aceptado CONACEM, esperando la sanción legal definitiva.

Palabras clave: Acreditación, certificación, cirugía, Chile.

La certificación y recertificación de especialistas médicos está de gran actualidad. En este campo, la Corporación Nacional Autónoma de Certificación de Especialidades Médicas (CONACEM) tiene un papel preponderante. Hasta 1984, en Chile sólo existía el reconocimiento de especialista por las Universidades con programas de formación acreditados por la Asociación de Facultades de Medicina de Chile (ASOFAMECH). Luego esta responsabilidad quedó radicada en CONACEM, corporación de derecho privado, fundada en 1984 y constituida por representantes de la Academia de Medicina del Instituto de Chile, Asociación de Facultades de Medicina de Chile, Colegio Médico de Chile A.G. y las Sociedades Científicas, integrando el directorio de la Corporación en proporción de 4 delegados cada cual, salvo la Academia de Medicina que tiene uno. El Ministerio de Salud aporta un representante con derecho a voz y existe un Secretario. Junto al Directorio trabajan 54 Comités de Especialidades con 7 miembros cada uno, con dos representantes de ASOFAMECH, Colegio Médico de Chile A.G. y de la Sociedad Científica pertinente. El Presidente es nominado por el Directorio. A este total de 378 médicos se agregan los profesores examinadores, lo que significa que más de 500 médicos aportan su mejor saber, con mística encomiable, y sin remuneración alguna. Lo referente a CONACEM puede ser consultado en la página web (www.conacem.cl).

CONACEM posee características que son importantes para una institución que aspira a ser certificadora nacional. Ellas son:

1) Representatividad, ya que su constitución incorpora los estamentos más prestigiados de la Medicina chilena en: academia, ciencia, asistencia y aspectos gremiales.

2) Experiencia probada en 28 años de trayectoria.

3) Reconocimiento. CONACEM ha certificado a 12.294 especialistas que voluntariamente lo han solicitado, lo que expresa el prestigio que goza ante los prestadores de salud, individuales o institucionales.

4) Mística. El hecho que 500 médicos trabajen por
CONACEM, sólo por la satisfacción moral de apoyar a la medicina chilena y a los pacientes, es un galardón difícil de emular.

5) Transparencia. Los datos del proceso de certificación son obtenibles electrónicamente, y también comunicados a la Intendencia de Prestadores Médicos.

6) Independencia, desarrollando su labor ajena a consideraciones políticas, religiosas u otras.

CONACEM está centrada en la certificación de especialistas médicos y en la definición de especialidades médicas. No corresponde a ella la certificación de los programas de formación para especialistas, lo que es legalmente responsabilidad de agencias certificadoras, donde destaca la Agencia de Acreditación de Programas y Centros formadores de Especialistas (APICE).

\section{¿Qué define a una especialidad y a un especialista médico?}

Especialidad es un campo del saber médico que comprende el conocimiento, las patologías y sus tratamientos en relación armónica. Especialista es aquel médico que domina el conocimiento, diagnóstico y tratamiento de las enfermedades de una disciplina reconocida como especialidad. En esta definición el vocablo "domina" marca el sentido de lo que es un especialista. La condición de especialista se abre también al campo de la investigación, docencia y divulgación científica.

\section{¿Qué tipos de especialidades se reconocen?}

Existen dos tipos de especialidades: a) Primarias, a cuya formación se puede acceder desde el inicio de la vida profesional, con el único requisito de ser médico cirujano. CONACEM certifica 26 especialidades primarias, entre ellas Cirugía General; b) Derivadas que requieren de la certificación previa como especialista en la disciplina primaria de la cual se originan. CONACEM reconoce 26 especialidades derivadas, de las cuales 6 tienen a Cirugía General como la especialidad primaria. 
Una categoría especial es la Mención, que no constituye una especialidad, pero sí reconoce y avala la dedicación y capacitación en un área de una especialidad.

\section{¿Cuáles son las vías para certificarse como especialista en CONACEM?}

Existen 3 opciones: 1) Haber aprobado un Programa Universitario de formación acreditado por ASOFAMECH, o APICE; 2) Por Formación en el Extranjero. Esta vía requiere que el Comité de la especialidad haga un análisis de homologación entre el programa cursado en el extranjero, y los aprobados y vigentes en Chile. Si hay concordancia el candidato deberá someterse a un examen teórico y, de ser este aprobado, a uno práctico; 3) Por Adiestramiento en Práctica. Como requisito general el postulante debe haber trabajado en la especialidad por mínimo 5 años, en jornada de al menos 22 horas semanales, y con ligazón legal en centros de complejidad acorde con la disciplina, los cuales deben contar al menos con dos especialistas certificados que avalen el aprendizaje del candidato. Cada especialidad tiene requisitos adicionales específicos. Los candidatos deben rendir ante CONACEM un examen teórico y, una vez aprobado éste, otro práctico.

Para definir una especialidad o subespecialidad CONACEM lo hace a requerimiento de algunos de sus socios (Academia de Medicina de Chile, Sociedades Científicas, ASOFAMECH, Colegio Médico de Chile A.G.) o de los miembros del Directorio. Los requisitos básicos para iniciar ese estudio son: a) que exista legalmente una sociedad científica activa dedicada al área en análisis; b) que exista un programa universitario de formación acreditado en esa disciplina; c) que se cuente con una masa crítica mínima de 20 médicos ejerciendo la especialidad; d) que la disciplina a reconocer esté en acuerdo a lo que es la tradición de la medicina chilena.

\section{¿Cuál es la situación legal del reconocimiento de especialistas?}

Hasta 1984 el reconocimiento lo hacían las universidades con programas acreditados de formación. En 1984 CONACEM inicia la certificación de especialistas. En febrero del 2004 se dictó la ley 19.937 de Autoridad Sanitaria que establece un sistema de certificación de especialistas y encomienda a los Ministerios de Salud y Educación confeccionar un reglamento para determinar las entidades examinadoras y las condiciones para que ellas sean autorizadas. El 6 noviembre de 2008 se dicta el Decreto Supremo $n^{\circ} 57$ que responde a esa ordenanza y define 24 especialidades médicas y 18 subespecialidades, fija los requisitos y procedimientos, señala las modalidades para otorgar la certificación, y define la vigencia de la certificación por un período entre 5 y 10 años. Además, crea un registro público de prestadores individuales. En atención a que el proceso organizativo sufría retraso un nuevo Decreto Supremo, dictado el 2010, prorrogó hasta el año 2012 ciertos aspectos transitorios del Decreto Supremo $\mathrm{n}^{\circ} 57$ y que tenían relación con las entidades certificadoras, las que podrían registrarse e iniciar sus actividades en ese tiempo de prórroga. Además, reconocía que las certificaciones vigentes a la fecha (2008) serían válidas por un plazo de 7 años. En esta prórroga incluía las certificaciones como especialistas otorgadas por las universidades y por CONACEM, los reconocimientos de desempeño en práctica de la especialidad y los médicos listados en los convenios de especialidad con FONASA. El hecho práctico de ese mandato legal es que a partir de enero de 2015 todas las certificaciones y reconocimientos vigentes caducan. A la fecha está avanzado el reconocimiento de CONACEM como entidad certificadora y recertificadora de especialistas médicos.

\section{¿Qué es certificar y recertificar?}

Según la Real Academia de la Lengua Española certificar es asegurar, afirmar y dar por cierto algo, en este caso la calidad de especialista. También es hacer constar por escrito una realidad de hecho por quien tenga fe pública o atribución para ello. No se aparta de esta definición la que hace el Decreto Supremo n ${ }^{\circ} 57$ que señala que es un "proceso en virtud del cual se reconoce que un prestador individual domina un cuerpo de conocimientos y /o experiencias relevantes en un determinado ámbito del trabajo asistencial, para el otorgamiento del correspondiente certificado. Recertificar es refrendar la certificación de modo que las constataciones hechas inicialmente mantengan valor y actualidad.

La recertificación requiere de una certificación previa y, por lo tanto, aquellos médicos incluidos en categorías reconocidas por la ley (desempeño en práctica, convenio con FONASA), deben, como primera instancia, certificarse.

Esta definición de especialista, corre el riesgo de ser banalizada. Minimizar o alivianar lo que es un verdadero especialista, muchas veces por la urgencia de la contingencia, implica un menoscabo a la medicina porque: a) rompe con la fe pública en los organismos de la sociedad encargados de certificar que lo que se dice es verdadero; b) soluciona parcialmente los problemas que se pretenden abordar; c) disminuye la esperada eficacia y eficiencia del especialista; d) aumenta los costos en tiempo y dinero. f) genera inequidad para con los médicos que tienen la calidad de especialistas respecto de aquellos que no la cumplen.

La definición laxa de lo que es una especialidad o 
subespecialidad tiene el riesgo de la fragmentación o "balcanización" del cuerpo de conocimientos de la especialidad. La mayor tendencia a que ello ocurra sucede cuando se desarrollan actividades médicas centradas en una tecnología de compleja operatividad.

\section{¿Qué vigencia tienen las certificaciones?}

Las universidades, cuando otorgaban el título de especialista, lo consideraron vitalicio. Para CONACEM las certificaciones previas a 2008 eran permanentes; después, en atención a la nueva legislación se extendieron por 10 años en primera instancia, para que las renovaciones lo fueran por 7 años. A partir de agosto de 2008 y hasta el 30 de junio de 2013 CONACEM ha certificado 126 especialistas, con vigencia de 10 años.

\section{¿Qué razones avalan la recertificación?}

Son variadas: a) complejidad creciente de los conocimientos, tecnologías, destrezas, y fuentes de información, con emergencia de nuevos campos del saber y del actuar médico, que deben acotarse; b) rápida obsolescencia del conocimiento médico y de sus paradigmas; c) divulgación amplia e instantánea de los avances mundiales en medicina; d) demanda de los usuarios por contar con medicina del más alto nivel y efectividad; e) necesidad de asegurar la calidad de las prestaciones médicas por parte de las instituciones prestadoras de salud, y de los propios médicos; f) creciente judicialización de las acciones médicas que requiere clarificar responsabilidades y responder al concepto jurídico de "temeridad o no temeridad médica"; g) cumplir con la Ley n 19.966 del Régimen General de Garantías en Salud, referentes al acceso, calidad y oportunidad de las acciones médicas.

\section{¿Qué ha sucedido con la Cirugía General mirada desde CONACEM?}

El proceso de certificación en Cirugía General se inició en 1985 y desde entonces un total de 1.242 médicos han solicitado su certificación, lográndolo 1.088 postulantes $(87,6 \%)$. Cirugía General, como especialidad primaria, es requisito para la certificación de 6 subespecialidades: Cirugía Plástica y Reparadora, Cirugía de Cabeza, Cuello y Maxilo Facial, Cirugía de Tórax y Cardiovascular, Cirugía Vascular Periférica, Coloproctología y Medicina Intensiva.

Inicialmente, se reconoció como especialistas a los Profesores universitarios de la disciplina, (142 en total), modalidad que fue eliminada el 2004. El $87 \%$ de los médicos que la utilizó lo hizo antes de 1989. El universo restante de 946 especialistas corresponde mayoritariamente $(72 \%)$ a Programas Universitarios acreditados.
A la fecha, están acreditados 13 programas de formación de 3 años de duración. El cupo global de alumnos del conjunto es de 70 vacantes por año. Del total, 9 programas se desarrollan en Santiago, y el resto en provincias (Valparaíso, Concepción, Temuco y Valdivia). La distribución geográfica de los alumnos es equilibrada (69\% Santiago y $31 \%$ provincias).

La vía de Adiestramiento en Práctica ha sido usada por el $23 \%$ de los especialistas y la de Formación en el Extranjero es mínima $(<6 \%)$ no sobrepasando 3 médicos por año.

El desglose quinquenal de las diferentes vías se presenta en la Tabla 1. El primer quinquenio se aleja de la tónica general dado que corresponde al inicio del proceso de certificación.

A partir de 1990, el promedio anual de postulaciones y certificaciones ha aumentado llegando a duplicarse; el tramo 2010-2012, coincidente con la nueva legislación de especialidades evidencia un quiebre en este ritmo ascendente cuya explicación es compleja, sin poder descartar un menor interés por la Cirugía General.

La Tabla 2 muestra características demográficas de los postulantes a certificación en cuanto a su procedencia geográfica y género. El origen capitalino ha sido mayoritario, y en el último trienio especialmente marcado; como explicación no puede aducirse una mayor oferta de programas formativos en Santiago.

Una situación distinta sucede con el género de los Cirujanos Generales. Hace 23 años la relación era de una doctora por cada 39 cirujanos varones. Como lo muestra la Tabla 2 esta proporción fue minimizándose hasta ser hoy de 5/1.

La Tabla 3 desglosa quinquenalmente el número de postulantes y de médicos que completaron exitosamente el proceso de certificación, lo que al inicio es mayor por la certificación de los Profesores Universitarios. El universo de médicos que no consiguió aprobar la certificación $(9,8 \%)$ se explica por causas como no reunir los requisitos de postulación, o, de tenerlos, no aceptar dar examen o fracasar en éste. A partir del año 2002 se incorpora en Cirugía General el examen escrito, que a la fecha han rendido 77 postulantes, que con las repeticiones totalizan 85 exámenes. Este ha sido un gran paso en la certificación, cumpliendo un justo rol seleccionador de los candidatos capacitados con $89,4 \%$ de aprobación cumplidos once años de vida del examen. Los candidatos fracasados en el examen tienen otras dos opciones, lo que explica la disparidad entre el número de certificaciones y de aprobaciones del examen. El buen rendimiento del examen escrito se expresa en la mínima tasa de rechazo del examen práctico posterior, que alcanza en igual período de once años a $12,5 \%$ (Tabla 4 ). 
Tabla 1. Número, promedio anual y distribución porcentual de las certificaciones de especialistas en Cirugía General, distribuidos por quinquenios, según, la vía usada

\begin{tabular}{|c|c|c|c|c|c|c|c|}
\hline Período & & PU & AP & $\mathbf{F E}$ & Subtotal & Prof. Univ. & Total \\
\hline $1985-1989$ & $\begin{array}{l}\mathrm{n} \\
\overline{\mathrm{X}} \text { año } \\
\%\end{array}$ & $\begin{array}{r}196,0 \\
39,2 \\
62,8\end{array}$ & $\begin{array}{r}110,0 \\
22,0 \\
35,3\end{array}$ & $\begin{array}{l}6,0 \\
1,2 \\
1,9\end{array}$ & 312,0 & 118 & 430 \\
\hline $1990-1994$ & $\begin{array}{l}\mathrm{n} \\
\overline{\mathrm{x}} \text { año } \\
\%\end{array}$ & $\begin{array}{l}72,0 \\
14,4 \\
79,1\end{array}$ & $\begin{array}{r}14,0 \\
2,8 \\
15,4\end{array}$ & $\begin{array}{l}5,0 \\
1,0 \\
5,5\end{array}$ & 91,0 & 14 & 105 \\
\hline 1995 - 1999 & $\begin{array}{l}\mathrm{n} \\
\overline{\mathrm{X}} \text { año } \\
\%\end{array}$ & $\begin{array}{l}61,0 \\
12,2 \\
60,4\end{array}$ & $\begin{array}{r}28,0 \\
5,6 \\
27,7\end{array}$ & $\begin{array}{r}12,0 \\
2,4 \\
11,9\end{array}$ & 101,0 & 3 & 104 \\
\hline $2000-2004$ & $\begin{array}{l}\mathrm{n} \\
\overline{\mathrm{x}} \text { año } \\
\%\end{array}$ & $\begin{array}{r}115,0 \\
23,0 \\
73,2\end{array}$ & $\begin{array}{r}22,0 \\
4,4 \\
14,0\end{array}$ & $\begin{array}{r}20,0 \\
4,0 \\
12,7\end{array}$ & 157,0 & 7 & 164 \\
\hline $2005-2009$ & $\begin{array}{l}\mathrm{n} \\
\overline{\mathrm{x}} \text { año } \\
\%\end{array}$ & $\begin{array}{r}173,0 \\
34,6 \\
81,6\end{array}$ & $\begin{array}{r}32,0 \\
6,4 \\
15,1\end{array}$ & $\begin{array}{l}7,0 \\
1,4 \\
3,3\end{array}$ & 212,0 & & 212 \\
\hline $2010-2012$ & $\begin{array}{l}\mathrm{n} \\
\overline{\mathrm{X}} \text { año } \\
\%\end{array}$ & $\begin{array}{l}59,0 \\
19,7 \\
80,8\end{array}$ & $\begin{array}{r}11,0 \\
3,7 \\
15,1\end{array}$ & $\begin{array}{l}3,0 \\
1,0 \\
4,1\end{array}$ & 73,0 & & 73 \\
\hline Totales & $\begin{array}{l}\mathrm{n} \\
\%\end{array}$ & $\begin{array}{r}676,0 \\
71,5\end{array}$ & $\begin{array}{r}217,0 \\
22,9\end{array}$ & $\begin{array}{r}53,0 \\
5,6\end{array}$ & $\begin{array}{l}946 \\
100\end{array}$ & 142 & 1.088 \\
\hline
\end{tabular}

PU: Programa Universitario; AP: Adiestramiento en Práctica: FE: Formación en el Extranjero; Prof. Univ.: Profesor Universitario.

Tabla 2. Origen y género de los médicos certificados en Cirugía General, distribuidos según quinquenios

\begin{tabular}{|ccc|}
\hline Período & Santiago/Provincia* & Hombre/Mujer \\
$1985-1989$ & $1,21 / 1$ & $39,20 / 1$ \\
$1990-1994$ & $1,41 / 1$ & $18,30 / 1$ \\
$1995-1999$ & $1,56 / 1$ & $13,50 / 1$ \\
$2000-2004$ & $1,72 / 1$ & $9,90 / 1$ \\
$2005-2009$ & $3,36 / 1$ & $5,60 / 1$ \\
$2010-2012$ & $5,25 / 1$ & $4,80 / 1$ \\
\hline
\end{tabular}

*Domicilio declarado al postular.
Tabla 3. Postulaciones y certificaciones en Cirugía General en el período 1985-2012

\begin{tabular}{|lccc|}
\hline $\begin{array}{l}\text { Período } \\
\text { Años }\end{array}$ & $\begin{array}{c}\text { Postulaciones } \\
\overline{\mathbf{x}} / \text { año }\end{array}$ & $\begin{array}{c}\text { Certificados } \\
\overline{\mathbf{x}} / \mathbf{a n ̃ o}\end{array}$ & $\begin{array}{c}\text { Aprobación } \\
\text { \% }\end{array}$ \\
\hline $1985-1989$ & 103 & 88,4 & 86 \\
$1990-1994$ & 22 & 23,2 & 105 \\
\hline $1995-1999$ & 25 & 17,4 & 70 \\
$2000-2004$ & 36 & 34,9 & 96 \\
$2005-2009$ & 47 & 43,7 & 94 \\
$2010-2012$ & 26 & 25,0 & 97 \\
\hline
\end{tabular}

Tabla 4. Resultado de Exámenes Escritos y Prácticos durante el período 2002-2012

\begin{tabular}{|llcccc|}
\hline & & Postulantes & Aprobados & Reprobados & Total \\
Examen escrito & $\mathrm{n}$ & 77 & 76,0 & 9,0 & 85 \\
& $\overline{\mathrm{x}}$ anual & & 7,6 & 0,8 & \\
& $\%$ & & 89,4 & 10,6 & \\
Examen práctico & $\mathrm{n}$ & 74 & 70,0 & 10,0 & 80 \\
& $\overline{\mathrm{x}}$ anual & & 6,4 & 0,9 & \\
& $\%$ & & 8,8 & 12,5 & \\
\hline
\end{tabular}




\section{¿Cómo tiene diseñado CONACEM el proceso de Recertificación?}

La recertificación impone requisitos generales comunes a todas las especialidades que son: a) Estar certificado como especialista por CONACEM; b) Estar ejerciendo activamente la especialidad; c) Detentar una trayectoria profesional éticamente intachable; d) Completar 100 puntos (créditos), con posterioridad a la última certificación, según la escala confeccionada por CONACEM (Tablas 5 y 6 ). Este reglamento enfatiza la Educación Continua, al considerarla como único requisito obligatorio con al menos el $40 \%$ de los créditos, y de calidad demostrable, con actividades controladas en asistencia y evaluadas formalmente.

Hay que presentar el curriculum vitae de los
Tabla 5. Origen de los créditos necesarios para la Recertificación en CONACEM (mínimo 100)

\begin{tabular}{|ll|}
\hline 1. Actividades asistenciales & Máximo 50\% \\
$\begin{array}{l}\text { 2. Actividades académicas } \\
\text { 3. Actividades de Educación Continua: }\end{array}$ & $\begin{array}{l}\text { Máximo } 50 \% \\
\text { Mínimo } 70 \%\end{array}$ \\
\hline 4. Otras actividades: & Máximo 40\% \\
\hline
\end{tabular}

últimos 10 años y cancelar los aranceles correspondientes.

Los candidatos que no alcancen puntaje o no deseen presentar la documentación requerida, tendrán derecho a solicitar evaluación teórica y práctica.

Tabla 6. Reglamento de Recertificación. Créditos según actividades

\begin{tabular}{|c|c|c|}
\hline \multicolumn{3}{|l|}{ 1. Actividades Asistenciales (máx. 50 puntos) } \\
\hline \multicolumn{2}{|c|}{$\begin{array}{l}\text { Trabajo con contrato o convenio en departamento o servicio asistencial clínico, público o } \\
\text { privado }\end{array}$} & 5/año \\
\hline \multicolumn{2}{|c|}{ Trabajo en consulta privada solamente (a evaluar por comité correspondiente) } & 3/año \\
\hline \multicolumn{3}{|l|}{ 2. Actividades Académicas (máx. 50 puntos) } \\
\hline Docente Universitario & $\begin{array}{l}\text { Profesor (cualquier categoría) } \\
\text { Instructor o Ayudante } \\
\text { Con asignación de docencia }\end{array}$ & $\begin{array}{l}\text { 10/año } \\
\text { 5/año } \\
\text { 2/año }\end{array}$ \\
\hline $\begin{array}{l}\text { Publicaciones en revistas con comité editorial, } \\
\text { nacionales o extranjeras }\end{array}$ & $\begin{array}{l}\text { Autor principal o líder del grupo } \\
\text { Coautor }\end{array}$ & $\begin{array}{r}10 \mathrm{c} / \mathrm{u} \\
5 \mathrm{c} / \mathrm{u}\end{array}$ \\
\hline Capítulos de libros & $\begin{array}{l}\text { Autor } \\
\text { Coautor }\end{array}$ & $\begin{array}{r}10 \mathrm{c} / \mathrm{u} \\
3 \mathrm{c} / \mathrm{u}\end{array}$ \\
\hline \multicolumn{2}{|l|}{ Director o Docente en curso de perfeccionamiento } & 5 por curso \\
\hline \multicolumn{2}{|l|}{ Conferencista o Integrante de Mesa Redonda en Congreso } & 5 por congreso \\
\hline \multicolumn{3}{|c|}{ 3. Actividades de Perfeccionamiento o de Educación Continua (máx. 70 / mín. 40) } \\
\hline \multicolumn{2}{|l|}{ Becas y cursos de perfeccionamiento } & 10 por mes (máximo 30) \\
\hline \multicolumn{2}{|c|}{ Asistencia certificada a cursos y congresos de la especialidad, nacionales y extranjeros } & $3 \mathrm{c} / \mathrm{u}$ \\
\hline $\begin{array}{l}\text { Asistencia certificada a cursos de perfeccionamiento } \\
\text { con más de } 10 \text { horas lectivas }\end{array}$ & $\begin{array}{l}\text { Con evaluación } \\
\text { Sin evaluación }\end{array}$ & $\begin{array}{l}6 \mathrm{c} / \mathrm{u} \\
3 \mathrm{c} / \mathrm{u}\end{array}$ \\
\hline $\begin{array}{l}\text { Presentación trabajos de la especialidad a congresos } \\
\text { nacionales o extranjeros }\end{array}$ & $\begin{array}{l}\text { Autor principal o líder de grupo } \\
\text { Coautor }\end{array}$ & $\begin{array}{l}3 \mathrm{c} / \mathrm{u} \\
2 \mathrm{c} / \mathrm{u}\end{array}$ \\
\hline \multicolumn{2}{|l|}{ Asistencia certificada a reuniones clínicas } & $\begin{array}{l}3 \text { por año } \\
(0,2 \text { puntos por reunión })\end{array}$ \\
\hline \multicolumn{3}{|l|}{ 4. Otras Actividades (máx. 40 puntos) } \\
\hline \multicolumn{2}{|c|}{ Jefatura de Servicio público por concurso o de Departamento universitario } & 10 por año \\
\hline \multicolumn{2}{|c|}{ Miembro del directorio o de comité científico de la sociedad científica pertinente } & 5 por una vez \\
\hline \multicolumn{2}{|c|}{ Miembro de comité editorial de publicación de la especialidad } & 5 por comité \\
\hline \multicolumn{2}{|l|}{ Miembro de sociedad científica de la especialidad } & 5 por sociedad (máximo 10$)$ \\
\hline \multicolumn{2}{|l|}{ Otras (a evaluar) } & 10 máximo \\
\hline
\end{tabular}


El proceso de recertificación está por empezar y dada su complejidad y magnitud se requerirá de un esfuerzo mancomunado de todas las instancias de la Medicina Nacional para que lo apoyen y de CONACEM para que lo lleve a afecto.

Mirando la medicina chilena y sus potencialidades, el nivel de desarrollo nacional y los cambios sociológicos y legales ocurridos, el resolver el déficit de especialistas, con diferencias entre especialidades, pero general, depende de tres pilares intrínsecos y uno relacionado. Lo intrínseco dice referencia con: 1) el impulso para perfeccionar y expandir los programas universitarios de formación acreditados o en vías de hacerlo y aumentar sus cupos; 2) el apoyo sostenido de la autoridad sanitaria en cuanto a proveer plazas, remuneraciones, estímulos y equipamiento tecnológico para fomentar la vía de certificación por adiestramiento en práctica; 3) el desarrollo con carácter orgánico, sostenido, controlado, evaluado, y de calidad probada, de programas de educación médica continua, presencial o a distancia. La obsolescencia progresiva de la competencia profesional es un síndrome universal con muchas formas subclínicas que pasan desapercibidas, y que en general hay reticencia a reconocerlas. La Educación Continua es, hasta ahora, el único tratamiento conocido para la obsolescencia del saber y del actuar.

El pilar restante, no ligado a las especialidades, pero con notable repercusión sobre ellas, es la calidad y cobertura de la medicina de atención primaria. Si ella es deficitaria, por número, eficiencia o ambas razones, la demanda de especialistas será enorme y la capacidad disponible de ellos sobrepasada. No puede haber desarrollo de especialidades sin el propio de la atención primaria.

\section{¿Qué trae el futuro inmediato a nuestra medicina chilena?}

Varios son los hechos que se vislumbran o ya están en escena demandando solución. Señalaré: 1) la creciente demanda de especialistas, ahora acentuada por los imperativos legales que requieren ese concurso; 2) Acortamiento de los programas de especialidades derivadas, con nuevas vías de ingreso a ellas; 3) Fortalecimiento de la vía de adiestramiento en práctica. El centro de gravedad de esta vía debería pasar desde el notable esfuerzo individual de cada candidato, a una condición de mayor ayuda y respaldo del sistema con organización nacional; 4) Trabajo cooperativo entre universidades para el desarrollo de programas complejos; 5) Crecientes solicitudes de certificación por médicos, chilenos o no, formados en el extranjero; 6) Aparición de nuevas especialidades o subespecialidades.

Cualquier solución al acuciante problema de la carencia de especialistas requiere del concurso de todos los actores de la medicina chilena. Relegar esta responsabilidad sólo a la autoridad sanitaria ha probado conseguir soluciones tardías y parciales e insatisfacciones crecientes.

Además del fortalecimiento de la atención primaria, una propuesta de solución al déficit de especialistas requiere: 1) Aumento de los programas universitarios acreditados, y sus cupos, manteniendo la calidad. Aunque lo anterior abre una solución sólo a más que mediano plazo, no hay razón para no empezar. Esta generación de especialistas debe constituir el factor multiplicador cuando asentados a lo largo del país, idealmente en parejas, con destinación geográfica conocida desde el inicio de su formación, puedan sustentar la formación por adiestramiento en práctica de otros médicos en sus lugares de destino; 2) La autoridad sanitaria debería estimular estas destinaciones con plazas de trabajo, facilidades de asentamiento, equipamiento médico y remuneraciones. Se iniciaría un círculo virtuoso interesando a profesionales a usar la vía de formación en práctica y rompería la limitante actual para que esos interesados no tengan que interrumpir por dos o tres años su vida familiar e intereses personales para radicarse lejos de su residencia en centros universitarios de formación; realista es que esa formación se haga trabajando en su ciudad de residencia, bajo la supervisión de dos especialistas acreditados.

Los candidatos para la vía de adiestramiento en práctica requieren el soporte de una educación continua eficaz: En este campo las Facultades de Medicina, el Colegio Médico de Chile A.G. y las Sociedades Científicas pueden contribuir substantivamente. En suma, una solución integral para la carencia de especialistas requiere el concurso de todas las potencialidades de la medicina chilena. Ellas existen y necesitan ser coordinadas y orientadas. No hacerlo merece más que una simple explicación. 